\title{
AN INVERSION INTEGRAL
}

\section{R. G. BUSCHMAN}

For an integral transformation which involves a Chebyshev polynomial in the kernel an inversion integral is known [4]. A similar problem involving a Legendre polynomial has been solved [1]. By somewhat different methods we demonstrate an inversion integral for an integral transformation in which the kernel involves a Gegenbauer polynomial, $C_{n}^{k / 2}(x)$, also known as ultraspherical polynomials, and thus includes the known cases for $k=0,1$ respectively, where we take the standardization $C_{n}^{0}(x)=\lim _{k \rightarrow 0} k^{-1} C_{n}^{k}(x)$. We write, for integers $k$ and $n$ with $0<k<n$,

$$
\begin{aligned}
F_{n}^{k}(x)= & 2^{(k-1) / 2} \pi^{-1 / 2} \Gamma(k / 2) n ! \\
& \cdot[\Gamma(n+k)]^{-1}\left(x^{2}-1\right)^{(k-1) / 2} C_{n}^{k / 2}(x), \\
G_{n}^{k}(x)= & 2^{(k-1) / 2} \pi^{-1 / 2} \Gamma(k / 2)(n-k-1) ! \\
& \cdot[\Gamma(n-1)]^{-1}\left(1-x^{2}\right)^{(k-1) / 2} C_{n-k-1}^{k / 2}(x),
\end{aligned}
$$

and from this standardization for $k=0$ we have

$$
\begin{aligned}
& F_{n}^{0}(x)=(2 / \pi)^{1 / 2}\left(x^{2}-1\right)^{-1 / 2} T_{n}(x) \\
& G_{n}^{0}(x)=(2 / \pi)^{1 / 2}\left(1-x^{2}\right)^{-1 / 2} T_{n-1}(x)
\end{aligned}
$$

If $f^{(k+1)}(x)$ is sectionally continuous for $0<a \leqq x \leqq 1$ and $f^{(m)}(1)=0$ for $0 \leqq m \leqq k$, then

$$
\int_{x}^{1} F_{n}^{k}(t / x) g(t) d t=f(x)
$$

has the solution

$$
g(t)=\int_{t}^{1} G_{n}^{k}(t / y) y^{-n+k+1}\left(-y^{-1} d / d y\right)^{k+1}\left[y^{n+k-1} f(y)\right] d y
$$

for $0<a \leqq t \leqq 1$.

Since the integral is in the form of a convolution with respect to the Mellin transformation, formal application of this transformation, use of tables [3], and manipulation leads to the suggested form of the solution.

Received by the editors July 25, 1961 and, in revised form, August 28, 1961. 
The solution can be verified directly by first proving that for $u>1$

$$
J(u)=\int_{1 / u}^{1} F_{n}^{k}(v u) G_{n}^{k}(v) d v=\left(2^{k} k !\right)^{-1}\left(u^{2}-1\right)^{k} u^{n-k-1} .
$$

This integral can be rewritten in a standard form of a convolution for the Mellin transformation [5; or 3, 6.1(13)],

$$
\int_{0}^{\infty} F_{n}^{k}(v u) U(v u-1) G_{n}^{k}(v)[1-U(v-1)] d v,
$$

where $U(x)=0, x<0 ;=1, x>0$. Thus we have

$$
\mathfrak{N}\{J(u) ; s\}=\mathfrak{M}\left\{F_{n}^{k}(u) U(u-1) ; s\right\} \mathfrak{N}\left\{G_{n}^{k}(u)[1-U(u-1)] ; 1-s\right\} .
$$

From Rodrigues' formula $[2,10.9(11)]$ (noting that with the standardization for $k=0$ this reduces to $[3,10.11(14)])$ and the tables $[3$, $6.2(32), 6.1(10)]$ and after some simplification

$$
\begin{aligned}
& \operatorname{Tr}\left\{F_{n}^{k}(u) U(u-1) ; s\right\} \\
& \quad=\left(2^{k+1} \pi\right)^{-1 / 2} \Gamma[(1-s+n) / 2] \Gamma[(1-k-n-s) / 2]\left[2^{s} \Gamma(1-s)\right]^{-1} \\
& \operatorname{Tr}\left\{G_{n}^{k}(u)[1-U(u-1)] ; s\right\} \\
& \quad=\pi^{1 / 2} 2^{-s-(k-1) / 2} \Gamma(s)(\Gamma[(2+s-n+k) / 2] \Gamma[(s+n) / 2])^{-1},
\end{aligned}
$$

so that

$$
\mathfrak{T}\{J(u) ; s\}=\left(2^{k+1} k !\right)^{-1} B[(1-s-n-k) / 2, k+1] .
$$

But also from the tables $[3,6.2(32), 6.1(7)]$

$$
\left(2^{k} k !\right)^{-1}\left(u^{2}-1\right)^{k} u^{n-k-1} U(u-1)
$$

has the same Mellin transform, hence the formula follows.

Consider the iterated integral

$$
I(x)=\int_{x}^{1} F_{n}^{k}(t / x)\left(\int_{t}^{1} G_{n}^{k}(t / y) y^{-n+k+1}\left(-y^{-1} d / d y\right)^{k+1}\left[y^{n+k-1} f(y)\right] d y\right) d t
$$

which is formed by direct substitution of the proposed value for $g(t)$ into the integral equation. If the order of integration is changed

$$
I(x)=\int_{x}^{1} y^{-n+k+1}\left(-y^{-1} d / d y\right)^{k+1}\left[y^{n+k-1} f(y)\right]\left(\int_{x}^{y} F_{n}^{k}(t / x) G_{n}^{k}(t / y) d t\right) d y .
$$

Thus if we let $v=t / y, u=y / x$ the inner integral becomes 


$$
y J(u)=\left(2^{k} k !\right)^{-1}\left(y^{2}-x^{2}\right)^{k} y^{n-k} x^{-n-k+1},
$$

and we can write

$$
I(x)=-\left(2^{k} k !\right)^{-1} x^{-n-k+1} \int_{x}^{1}\left(y^{2}-x^{2}\right)^{k} d\left\{\left(-y^{-1} d / d y\right)^{k}\left[y^{n+k-1} f(y)\right]\right\} .
$$

Successive integrations by parts and application of the conditions $f^{(m)}(1)=0$ then yields $I(x)=f(x)$.

\section{REFERENCES}

1. R. G. Buschman, $A n$ inversion integral for a Legendre transformation, Amer. Math. Monthly 69 (1962), 288-289.

2. A. Erdélyi et al., Higher transcendental functions, Vol. 2, McGraw-Hill, New York, 1953.

3. - Tables of integral transforms, Vol. 1, McGraw-Hill, New York, 1954.

4. Ta-Li, $A$ new class of integral transforms, Proc. Amer. Math. Soc. 11 (1960), 290298.

5. E. C. Titchmarsh, Introduction to the theory of Fourier integrals, 2nd ed., University Press, Oxford, 1959.

Oregon State University 